\title{
Erratum to: Universal Health Coverage in Massachusetts Did Not Change the Trajectory of Arthroplasty Use or Costs
}

\author{
Steven M. Kurtz PhD, Edmund Lau MS, Kevin L. Ong PhD, \\ Jeffrey N. Katz MD, Kevin J. Bozic MD, MBA
}

Published online: 25 October 2016

(C) The Association of Bone and Joint Surgeons( 2016

\section{Erratum to: Clin Orthop Relat Res (2016) \\ 474:1090-1098 \\ DOI 10.1007/s11999-015-4643-9}

Our study, "Universal Health Coverage in Massachusetts Did Not Change the Trajectory of Arthroplasty Use or Costs" published in Clinical Orthopaedics and Related Research $^{\mathbb{R}}$ is an analysis of the State Inpatient Database (SID), a restricted-access, publicly available dataset that is maintained by the Agency for Healthcare Research and Quality (AHRQ). One of the provisions of the SID Data Use Agreement (DUA), which we signed when purchasing the data, is that no data observation involving less than or equal to 10 observations is to be published. This measure was put in place by AHRQ to protect individual patients' privacy and to prevent the potential disclosure of personal information. In our manuscript, there are some tabulated cells in Table 1 that are in violation of this provision. This represents an oversight on my part and an inadvertent failure to comply with the DUA. For this, we apologize. We have provided a revised Table 1.

While I am fully confident that nothing in our paper could be used to identify individuals or their personal information, the fact remains that the disclosures are in violation of the AHRQ guidelines. We have therefore asked the editors of Clinical Orthopaedics and Related Research $^{\mathbb{R}}$ to post an erratum that is in compliance with the AHRQ guidelines, and request that, going forward, authors cite the corrected version of our manuscript.

The online version of the original article can be found under doi:10.1007/s11999-015-4643-9.

S. M. Kurtz, K. L. Ong

Exponent, Inc, Philadelphia, PA, USA

E. Lau

Exponent, Inc, Menlo Park, CA, USA

J. N. Katz

Harvard Medical School, Boston, MA, USA

K. J. Bozic

Dell Medical School, The University of Texas at Austin, Austin,

TX, USA

S. M. Kurtz ( $\square)$

3401 Market Street, Suite 600, Philadelphia, PA 19104, USA

e-mail: skurtz@exponent.com 
Table 1. THAs and TKAs reimbursed by payer

\begin{tabular}{|c|c|c|c|c|c|c|c|c|c|c|c|c|}
\hline $\begin{array}{l}\text { Payer } \\
\text { category }\end{array}$ & Primary payer & 2002 & 2003 & 2004 & 2005 & 2006 & 2007 & 2008 & 2009 & 2010 & 2011 & Total \\
\hline \multirow[t]{5}{*}{ Other } & Commonwealth care & $*$ & $*$ & $*$ & $*$ & $*$ & 73 & 183 & 229 & 266 & 343 & 1095 \\
\hline & Health safety net $(2009+)$ & $*$ & $*$ & $*$ & $*$ & $*$ & $*$ & $*$ & 13 & 41 & 38 & 92 \\
\hline & Other government payment & 47 & 66 & 91 & 86 & 82 & 90 & 83 & 92 & 97 & 93 & 827 \\
\hline & Other nonmanaged care plan & 18 & 17 & 11 & 15 & 18 & $*$ & 34 & $*$ & $*$ & $*$ & 123 \\
\hline & Worker compensation plan & 133 & 123 & 150 & 162 & 170 & 192 & 212 & 211 & 215 & 215 & 1783 \\
\hline No charge & Free care & 69 & 84 & 81 & 91 & 109 & 56 & 37 & 25 & $*$ & * & 559 \\
\hline Self-pay & Self-pay & 16 & 20 & 16 & 22 & 13 & 21 & 21 & $*$ & 19 & 14 & 169 \\
\hline \multirow[t]{9}{*}{ Private } & Auto insurance plan & $*$ & $*$ & $*$ & $*$ & $*$ & $*$ & $*$ & $*$ & $*$ & $*$ & 35 \\
\hline & Blue Cross & 509 & 604 & 696 & 703 & 764 & 853 & 828 & 822 & 856 & 851 & 7486 \\
\hline & Blue Cross, managed care & 1417 & 1632 & 1995 & 2337 & 2701 & 2909 & 2978 & 3391 & 3403 & 3345 & 26,108 \\
\hline & Commercial & 429 & 480 & 518 & 580 & 600 & 649 & 679 & 662 & 726 & 845 & 6168 \\
\hline & Commercial, managed care & 169 & 181 & 220 & 223 & 267 & 239 & 280 & 311 & 347 & 441 & 2678 \\
\hline & Exclusive provider plan & $*$ & $*$ & $*$ & $*$ & 12 & 24 & $*$ & 12 & 12 & * & 90 \\
\hline & Health maintenance organization & 1750 & 1957 & 2033 & 2114 & 2131 & 2312 & 2457 & 2787 & 3123 & 3428 & 24,092 \\
\hline & $\begin{array}{l}\text { Other preferred provider organization } \\
\text { and managed care }\end{array}$ & 294 & 329 & 360 & 346 & 337 & 389 & 476 & 453 & 395 & 403 & 3782 \\
\hline & Point of service plan & $*$ & 23 & 58 & 37 & 62 & 53 & 49 & 65 & 100 & 71 & 521 \\
\hline \multirow[t]{2}{*}{ Medicaid } & Medicaid & 319 & 338 & 371 & 369 & 407 & 444 & 445 & 501 & 529 & 497 & 4220 \\
\hline & Medicaid, managed care & 93 & 115 & 150 & 205 & 221 & 281 & 348 & 372 & 386 & 463 & 2634 \\
\hline \multirow[t]{2}{*}{ Medicare } & Medicare & 5959 & 7062 & 7692 & 7865 & 8134 & 8387 & 8439 & 8560 & 9165 & 9468 & 80,731 \\
\hline & Medicare, managed care & 1978 & 1724 & 1655 & 1808 & 1827 & 1894 & 2102 & 2211 & 2453 & 2138 & 19,790 \\
\hline Unknown & Missing/unknown & $*$ & $*$ & $*$ & $*$ & $*$ & $*$ & $*$ & $*$ & $*$ & $*$ & 13 \\
\hline Total & & 13,207 & 14,765 & 16,105 & 16,972 & 17,858 & 18,876 & 19,665 & 20,733 & 22,143 & 22,672 & 182,996 \\
\hline
\end{tabular}

$*<10$ Patients. 